\title{
La reforma penitenciaria será con los funcionarios o no será. Acerca de la necesidad de contar con el personal para lograr el cambio en la institución penitenciaria
}

\section{Cristina Güerri}

Universitat Pompeu Fabra. Grupo de Investigación en Criminología y Sistema Penal cristina.guerri@upf.edu

\section{Resumen}

Este texto pertenece a una mesa redonda que ofrece un debate con James B. Jacobs, autor del clásico de la sociología de las prisiones Stateville: The penitentiary in mass society, sobre la vigencia de su obra cuarenta años después. Este comentario se centra en la importancia de la moral laboral del personal penitenciario para el buen funcionamiento de la prisión y el éxito de las reformas que se deseen implementar. En primer lugar, se reproduce la tesis de Jacobs en Stateville respecto a esta cuestión. A continuación, se argumenta que dicho planteamiento es también aplicable a la realidad penitenciaria española. Finalmente, se señalan las implicaciones de todo ello para el desarrollo y la implementación de nuevas políticas penitenciarias.

Palabras clave: funcionarios penitenciarios; reforma penitenciaria; moral laboral

Abstract. Prison reform with or without correctional officers: The importance of prison staff in bringing about change in penitentiary institutions

This text forms part of a round table discussion with James B. Jacobs, author of the classic study on the sociology of prisons Stateville: The Penitentiary in Mass Society, and examines the validity of his work 40 years later. The commentary addresses the importance of prison officer morale in the functioning of penitentiary institutions. Firstly, the thesis Jacob presents in Stateville on this issue is reproduced. It is then argued that his reasoning is also relevant to the Spanish penitentiary context. To conclude, the implications for the design and implementation of new penitentiary policies are discussed.

Keywords: prison officers; prison reform; job morale 


\section{Sumario}

$\begin{aligned} \text { 1. Las consecuencias de } & \text { 2. El funcionario de interior en } \\ \text { la desmoralización de los guardias para } & \text { la reforma penitenciaria española } \\ \text { el buen funcionamiento de la institución } & \text { 3. La política penitenciaria } \\ \text { penitenciaria } & \text { y el funcionario de prisiones } \\ & \text { Referencias bibliográficas }\end{aligned}$

Una de las virtudes más señaladas de Stateville es el haber concebido la prisión como una organización conectada a la realidad que la rodea y haber mostrado cómo los cambios sociales, legales y políticos del mundo exterior se filtran a través de sus muros y la transforman (ver, por ejemplo, Crewe, 2016). Sin embargo, pensar la prisión como una organización también permitió a Jacobs incorporar aspectos como la gestión efectuada por la dirección o la realidad laboral del personal penitenciario al análisis de la prisión, en vez de limitarse a examinar la vida de los internos como había hecho gran parte de la literatura previa (Jacobs, 2018).

Una de las aportaciones que realiza el autor desde esta perspectiva es resaltar la importancia de la moral laboral de los guardias para el buen funcionamiento de las prisiones, cuestión a la que dedicaremos la presente reflexión. En primer lugar, se presentarán las razones por las cuales Jacobs, a raíz de lo sucedido en Stateville, defiende tal posición. Posteriormente, se mostrará que tal planteamiento es también aplicable a la realidad penitenciaria española. El artículo concluirá señalando las implicaciones que todo ello tiene para el desarrollo y la implementación de nuevas políticas penitenciarias.

\section{Las consecuencias de la desmoralización de los guardias para el buen funcionamiento de la institución penitenciaria}

La moral laboral del personal es importante en cualquier trabajo, puesto que influye en el grado de esfuerzo de los trabajadores (Weakliem y Frenkel, 2006). No obstante, la historia de Stateville muestra que este aspecto es de especial relevancia en el ámbito penitenciario, ya que «en prisión, si la moral del personal es baja [...] pueden suceder cosas terribles» (Jacobs, 2018).

Las transformaciones que tuvieron lugar en Stateville a partir de los años sesenta y que modificaron el funcionamiento de la institución también afectaron, lógicamente, a los guardias que trabajaban en ella (Jacobs, 1977). Primeramente, los medios a disposición de los guardias para ejercer el control y la disciplina se habían visto limitados por la supervisión judicial de sus actuaciones. Los tribunales tasaron los procedimientos que había que utilizar en cada situación, lo que diluyó la autoridad que los guardias habían ostentado hasta ese momento. Además, los internos no dudaban en recordar a los guardias las nuevas normas que había que aplicar cada vez que intentaban proceder a la antigua usanza, lo que les generaba una gran frustración. En segundo lugar, la prensa había dejado de ser una aliada de los administradores de la prisión 
para pasar a denunciar las malas condiciones de los internos, los cuales eran presentados como víctimas. Esta situación causaba desánimo en los guardias, puesto que, lejos de ser valorados socialmente por el trabajo que realizaban, eran representados como cómplices del sufrimiento de los internos. Por último, el cambio del perfil del interno que ingresaba en prisión y la reproducción de las bandas delictivas en el interior de la institución habían dificultado el mantenimiento del orden en el centro penitenciario. En conjunto, la situación había superado a los guardias, quienes empezaron a sentir que su mundo se desmoronaba a su alrededor y se veían incapaces de reaccionar (Jacobs, 1977).

En tal situación, un administrador conocedor de los problemas de sus empleados hubiera sido capaz de detectar la desmoralización de los guardias y actuar en consecuencia. De acuerdo con Jacobs (2018), la moral laboral de los funcionarios penitenciarios puede mejorar si quien dirige la prisión muestra al personal que se le toma en consideración. Esto puede hacerse de muchas maneras: desde hablar con los funcionarios para conocer sus preocupaciones hasta mejorar las condiciones de retribución y promoción laboral, pasando por proporcionarles un espacio de descanso dedicado exclusivamente al personal. De hecho, investigaciones hechas en correccionales muestran que factores organizativos como los que Jacobs señala tienen una gran influencia en la moral de los funcionarios (Minor et al., 2014). No obstante, la actuación de la dirección de Stateville en la situación anteriormente descrita fue totalmente distinta.

Tras la dimisión del antiguo director, y en el marco del proceso de burocratización de la institución penitenciaria, se nombró a un nuevo administrador de perfil profesional. Influido por las corrientes rehabilitadoras, el nuevo director manifestó, nada más llegar al cargo y ante todos los internos, que trabajaría por y para ellos, con lo que puso rápidamente a todos los guardias en su contra. El resto de su gestión tampoco contribuyó a mejorar su relación con este grupo del personal. Por ejemplo, para la toma de decisiones buscó asesoramiento en el personal civil de la prisión, sin consultar nunca a los guardias. Asimismo, les retiró la potestad de reducir a los internos y llevarlos a aislamiento si no disponían de la aprobación de un superior, lo que fue interpretado como un ataque a su autoridad. Todo ello, lejos de animar a los guardias, ahondó en su desmoralización: su trabajo era difícil y mal pagado, cada vez disponían de menos autonomía para realizarlo y no contaban con el apoyo de sus superiores. En consecuencia, los guardias hacían cada vez peor su trabajo, la seguridad disminuía, la violencia escalaba y ni siquiera las tareas más rutinarias se completaban de forma exitosa. Stateville se encontraba sumida en el caos (Jacobs, 1977).

La inestabilidad generada fue tal, que la dirección fue relevada y el propio subdirector del Department of Corrections tomó el mando. El nuevo administrador abordó la situación mediante una política basada en la detección y solución de problemas, en cuya implementación contó también con la opinión de los guardias de Stateville, representados por sus lugartenientes. En poco tiempo, el centro recuperó la estabilidad (Jacobs, 1977). Probablemente por ello Jacobs (2018) afirma que su consejo para cualquiera que asumiera la dirección de una prisión sería que, nada más llegar, hablara con su personal, les 
preguntara por sus preocupaciones, escuchara sus propuestas y les demostrara que se toma los problemas que les afectan muy, muy en serio.

\section{El funcionario de interior en la reforma penitenciaria española}

Si trasladamos esta cuestión a la realidad penitenciaria española, observamos que la posición de Jacobs fue también la de pensadores y prácticos de nuestro país. El correccionalismo español del siglo XIX había batallado por una ejecución de las penas privativas de libertad más humana y orientada a la reforma del penado, pero siempre consciente de que el elemento clave para llevar a cabo tal reforma era el personal. Por ejemplo, Lastres (1887: 177) manifestaba: «La base del sistema penal no son los edificios [...] la reforma descansa, no en los edificios, sino en el personal» (citado en García Valdés, 2006).

En relación con la concesión de importancia al personal por nuestros prácticos, encontramos un ejemplo excelente en la historia reciente de España. Durante la posguerra, el sistema penitenciario español funcionaba como una burocracia clásica en la que el régimen predominaba sobre el tratamiento. Los funcionarios de vigilancia tenían una posición fuerte dentro de la prisión, puesto que la aplicación de castigos y recompensas dependía en buena medida de ellos. De hecho, se solía decir que llevaban la prisión con la gorra, porque la mera visión de su gorra, símbolo de la autoridad del funcionario, servía para apaciguar los ánimos de los internos. Sin embargo, las modificaciones introducidas en el Reglamento de los Servicios de Prisiones en los años sesenta cambiaron la situación, con consecuencias negativas para el orden en la prisión y la estabilidad laboral de los funcionarios de vigilancia (Caballero, 1981).

La entrada de la ideología rehabilitadora en prisión fue una de las corrientes que transformaron las instituciones penitenciarias y, en consecuencia, la situación de sus guardias (Crouch, 1980). En Europa, las Reglas Mínimas para el Tratamiento de los Reclusos aprobadas en Ginebra en 1955 afirmaron la rehabilitación del condenado por medio del tratamiento como una de las finalidades que debía perseguir la privación de libertad, idea que empezó a calar en la doctrina española y se plasmó en nuestro ordenamiento jurídico a finales de los años sesenta introduciendo, primero, criterios de individualización científica del tratamiento e incorporando, después, al Cuerpo de Prisiones a los especialistas que debían aplicar estas nuevas técnicas de observación y tratamiento (Roldán, 1988).

Estos cambios tuvieron una gran repercusión en los funcionarios de vigilancia. Por una parte, la reconceptualización de las recompensas como parte del tratamiento debilitó su posición ante los internos. Por la otra, la posibilidad de que el nuevo personal técnico optara a los puestos de mando disminuyó sus expectativas de progresión laboral. En consecuencia, los funcionarios de vigilancia comenzaron a sentirse desmoralizados justo en el momento en que la conflictividad penitenciaria iba en aumento, conflictividad que alcanzaría su zénit con los motines expresivos que perpetrarían los presos comunes tras 
quedar excluidos de las dos amnistías políticas aprobadas durante la transición (Caballero, 1981). Carlos García Valdés, penitenciarista al que se encargó la redacción de la nueva ley penitenciaria y director general durante este período, lo relata del siguiente modo: «[E]n dos rasgos definidores se resume la situación anímica de los protagonistas de la relación carcelaria: presos enormemente crecidos y funcionarios aplomados» (García Valdés, 1978: 95). Y precisamente los funcionarios fueron uno de los ejes en los que basó la reforma penitenciaria que impulsó desde la Dirección General de Instituciones Penitenciarias y con la que consiguió recuperar la normalidad en el ámbito penitenciario:

[M]i preocupación máxima fueron los funcionarios, globalmente considerados; 'con ellos — me decían mis amigos el Inspector y el Subinspector General penitenciarios- no tendrás problemas, siempre que les expliques en qué consiste la reforma y te confíes a ellos': y es exacto; no sólo me han demostrado ser un Cuerpo disciplinado, sino que he comprobado cómo desean la reforma penitenciaria. Y yo me he volcado con ellos: les he hablado y escuchado como nadie hasta ahora lo había hecho, defendido inequívocamente ante el Congreso, reiterado mi apoyo incondicional en momentos de desánimo y, sobre todo, les he apoyado en algo que hacía años reclamaban: su derecho a constituir una asociación profesional y apolítica. Hoy ambos nos necesitamos recíprocamente y, si cabe, soy yo quien más de los mismos precisa (García Valdés, 1978: 98-99).

De manera similar a lo que había sucedido en Stateville, las nuevas corrientes ideológicas que habían modificado la forma de pensar y organizar las prisiones en España y los cambios políticos que acontecieron habían comprometido la estabilidad del sistema penitenciario español y mermado la moral del personal penitenciario. Y, también del mismo modo, la sensibilidad de una administración que supo valorar la importancia del personal para lograr el cambio y consiguió su compromiso con este resultó clave para recuperar la estabilidad y emprender la reforma deseada.

\section{La política penitenciaria y el funcionario de prisiones}

El objetivo de este texto ha sido enfatizar la importancia que tiene para la estabilidad y el buen funcionamiento de las prisiones el hecho de que desde la administración penitenciaria se tenga presente al funcionario en todo momento. Los ejemplos aquí desarrollados ilustran que el buen funcionamiento de la institución penitenciaria se ve perjudicado cuando la realidad laboral de la prisión sufre cambios sin que los funcionarios reciban apoyo de la administración para afrontarlos.

Tal afirmación también es aplicable para aquellos casos en los que la iniciativa transformadora forma parte de la política penitenciaria implementada por la administración. Por ejemplo, Thomas (1972) analiza la evolución del rol del funcionario de prisiones inglés desde 1850 hasta 1970 y muestra que los diferentes intentos de reforma llevados a cabo en dicho período generaron alienación en los funcionarios al excluirlos de su implementación, lo que dio 
lugar a un incesante conflicto entre dicho colectivo y la administración penitenciaria, que impidió que las políticas promovidas tuvieran el efecto deseado. De manera similar, Kauffman (1988) extrae de su investigación en la prisión de Walpole (Massachusetts) que muchos intentos de reformar las prisiones se han visto malogrados por la incapacidad de la administración penitenciaria de incorporar los problemas y las perspectivas de los guardias. También Lin (2002) demuestra que incluso los programas mejor diseñados fracasarán si en su aplicación no cuentan con la complicidad del personal penitenciario. Finalmente, Lombardo (1989) relata que los funcionarios de la prisión de Auburn adoptaron prácticas características del sabotaje industrial para condenar al fracaso ciertas políticas que habían sido aprobadas sin su beneplácito. En conjunto, todas estas investigaciones desaconsejan el impulso de medidas que no tengan el apoyo de los funcionarios penitenciarios: la imposición de una reforma a pesar de los funcionarios difícilmente funcionará.

Con ello no se quiere decir que se deba renunciar a desarrollar nuevas políticas si no se cuenta con la conformidad absoluta de todo el personal, pero sí que se debería intentar contar con su perspectiva en lugar de tratar de imponer las reformas asumiendo que los funcionarios, como último escalafón de la jerarquía penitenciaria, seguirán la cadena de mando y cumplirán las órdenes que reciban. El funcionario es quien, en última instancia, aplica la política penitenciaria y le da forma, y por ello se le debe hacer partícipe de la reforma. Incluir a los funcionarios en el desarrollo, la implementación y la evaluación de las políticas contribuye a que estos se sientan valorados, lo que aumenta su moral y su desempeño laboral; a detectar problemas y proponer soluciones; en definitiva, a un mejor funcionamiento de la institución penitenciaria.

Todo lo señalado nos lleva a concluir que el compromiso del personal penitenciario es un elemento irrenunciable para la consecución de cualquier cambio en la institución penitenciaria. Y en este mensaje hemos querido insistir, puesto que, como dice Jacobs (2018), «a veces no es fácil para los activistas» ni, me atrevería a añadir, para los académicos, «darse cuenta de que, para que las condiciones de los internos mejoren, primero deben ser buenas para el personal». $\mathrm{O}$, expresado con otras palabras: la reforma penitenciaria será con los funcionarios o no será.

\section{Referencias bibliográficas}

CABAllero, Juan José (1981). «La conflictividad en las prisiones españolas: una perspectiva histórica y sociológica». Revista de Estudios Penitenciarios, 121-68.

CREWE, Ben (2016). "The sociology of imprisonment». En: Yvonne Jewkes, Ben Crewe y Jamie Bennett (eds.). Handbook on Prisons. 2a ed. Londres: Routledge, p. 77-100.

Crouch, Ben M. (1980). «The guard in a changing prison world». En: Ben M. Crouch (ed.). The keepers: Prison guards and contemporary corrections. Springfield: Charles C. Thomas, 5-45.

García VAldés, Carlos (1978). «La reforma penitenciaria española». Estudios Penales y Criminológicos, 2, 91-104. 
- (2006). La ideología correccional de la reforma penitenciaria española del siglo XIX. Madrid: Edisofer.

JACOBS, James B. (1977). Stateville: The penitentiary in mass society. Chicago: University of Chicago Press.

- (2018). "Author meets critics: Stateville Revisited». III Conferencia Internacional del Máster en Criminología y Ejecución Penal. Barcelona, 11 de mayo.

Kauffman, Kelsey (1988). Prison officers and their world. Cambridge: Harvard University Press.

LASTRES, Francisco (1887). Estudios penitenciarios. Madrid: Establecimiento Tipográfico de Pedro Núñez.

LIN, Ann Chih (2002). Reform in the making: The implementation of Social Policy in Prison. Princeton: Princeton University Press.

LOMBARDO, Lucien X. (1989). Guards imprisoned: correctional officers at work. 2a ed. Nueva York: Elsevier.

Minor, Kevin I.; Wells, James B.; Lambert, Eric G. y Keller, Peggy (2014). «Personal and work environment antecedents of job moral among staff in juvenile corrections». Criminal Justice and Behavior, 41 (11), 1.308-26. <https://doi.org/10.1177/0093854814544702>

Roldán, Horacio (1988). Historia de la prisión en España. Barcelona: PPU.

Thomas, James E. (1972). The English prison officer since 1850: A study in conflict. Londres: Routledge \& Kegan Paul Books.

WeAkliem, David L. y Frenkel, Stephen J. (2006). «Morale and workplace performance». Work and Occupations, 33 (3), 335-61. <https://doi.org/10.1177/0730888406290054> 
